\title{
A computer simulation of the effect of temperature on melt chain dimensions of random short chain branched polyethylene
}

\author{
Javier Ramos *, Juan F. Vega, Javier Martínez-Salazar \\ Biophym, Departamento de Física Macromolecular, Instituto de Estructura de la Materia (IEM-CSIC) c/Serrano 113 bis, 28006 Madrid, Spain
}

\section{A R T I C L E I N F O}

\section{Keywords:}

Random copolymers

Molecular dynamics

Short chain branch

Conformational analysis

\begin{abstract}
A B S T R A C T
It is well established that the chain dimensions of a polymer are intimately related to the viscoelastic fingerprint and the relevant macroscopic properties of the material such as the entanglement modulus and its melt viscosity. In this work, the chain properties have been obtained by means of molecular dynamics simulations computed at different temperatures in a series of ethylene/1-butene copolymers with constant number of carbons in the backbone but varying branch content, from linear polyethylene to poly(1-butene) with a range of molecular weight extending from $7012 \mathrm{~g} / \mathrm{mol}$ till $14025 \mathrm{~g} / \mathrm{mol}$. The simulations were performed in a time window up to 5 $\mu$ s at four different temperatures between 450 and $600 \mathrm{~K}$. Simulation results are in good agreement with previous SANS experiments performed in this kind of model polymers. The influence of the amount of ethyl branches on the temperature dependence of the backbone conformation was analyzed. Thus, the observed trends of chain dimensions with increasing SCB content and temperature can be explained by the variation in content of the different trans-trans, gauche-trans and gauche-gauche dyads along the polymer backbone.
\end{abstract}

\section{Introduction}

Macromolecular chain dimension is a key factor of polymers, as it is directly related to physical properties, entanglement state and melt viscosity [1] [-] [4]. In particular, this parameter plays an important role while modeling polymeric materials since it influences the development of force fields in both atomistic and coarse-grained methods. One of the most interesting case-study for such computer simulations is the polyethylene (PE) family, as it contains the simplest chemical polymeric chains. There are several factors related to the molecular architecture that have an impact on the solid and melt physical properties of PE, being the amount of short chain branches (SCB) a relevant factor among them. However most of these studies have been restricted to models with relatively low SCB content-up to $40 \%$ of comonomer and focused on the study of static properties [5].

In this context, the dependence on the temperature of the macromolecular dimensions is given by the parameter by $\kappa=d\left[\ln \left\langle\operatorname{Rg}^{2}\right\rangle\right] / \mathrm{dT}$. This parameter can be obtained by measurements carried out in diluted solutions in theta conditions and also in the melt by means of thermoelastic experiments and Small Angle Neutron Scattering (SANS) [6]. The results obtained for $\kappa$ by Monte Carlo (MC) and Molecular Dynamics (MD) have been found to be in almost perfect agreement with the experiments in the case of linear PE, especially in simulations carried out with the TraPPE force field [7-10]. The effect of SCB in $\kappa$ has not been explored yet from the point of view of atomistic simulations, although experimental results from Fetters et al. on the variance of $\kappa$ obtained by SANS in a family of model ethylene/1-butene copolymers are available since 1997 [6]. An interesting result obtained by these authors was the change in $\kappa$ values, from negative to positive, as SCB content increased. The objective of our work is then to specifically study the chain dimensions and their temperature dependence as a function of the ethyl branch content is a series of model ethylene/1-butene random copolymers.

\section{Methods}

The linear polyethylene and the ethylene/1-butene chains of 250 monomers (Scheme 1) models-named as PEB-X used in the simulations are shown in Table 1 (X stands for the total number of ethyl branches per chain). Details concerning position of the branches and segment length distribution (spacers) between the branches are given in the Supplementary Information archive (Table S.1). All copolymer chains of a particular system are replicas having the same branch distribution (monomer distribution).

\footnotetext{
* Corresponding author. Instituto de Estructura de la Materia (IEM-CSIC), c/Serrano 113 bis, 28006, Madrid, Spain.

E-mail address: j.ramos@csic.es (J. Ramos).
} 

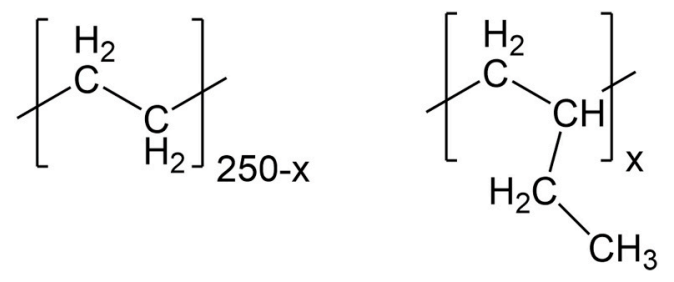

Scheme 1. Chemical structure of the copolymers studied.

Table 1

Simulated PEB-X models, branching content (ethyl/100C atoms), number of chains $\left(\mathrm{N}_{\text {chains }}\right)$, total particles $\left(\mathrm{N}_{\text {particles }}\right)$, molecular weight $\left(\mathrm{M}_{\mathrm{w}}\right)$ and simulated density $(\rho)$ and thermal expansion coefficient $(\alpha)$ at $450 \mathrm{~K}$.

\begin{tabular}{|c|c|c|c|c|c|c|}
\hline System & $\begin{array}{l}\text { Ethyl/ } \\
100 \mathrm{C} \\
\text { atoms }\end{array}$ & $\mathrm{N}_{\text {chains }}{ }^{\mathrm{a}}$ & $\mathrm{N}_{\text {particles }}$ & $\begin{array}{l}\mathrm{M}_{\mathrm{w}}(\mathrm{g} / \\
\mathrm{mol})\end{array}$ & $\begin{array}{l}\rho\left(\mathrm{g} / \mathrm{cm}^{3}\right) \\
\text { s.d. } \pm \\
0.002\end{array}$ & $\begin{array}{l}\alpha\left(10^{4}\right. \\
\left.\mathrm{K}^{-1}\right)^{\mathrm{c}}\end{array}$ \\
\hline $\begin{array}{l}\text { PEB- } \\
000\end{array}$ & 0 & 100 & 50000 & 7,012 & 0.760 & 8.36 \\
\hline $\begin{array}{l}\text { PEB- } \\
009\end{array}$ & 1.8 & 100 & 51800 & 7,265 & 0.761 & 8.34 \\
\hline $\begin{array}{l}\text { PEB- } \\
027\end{array}$ & 5.4 & 100 & 55400 & 7,770 & 0.763 & 8.36 \\
\hline $\begin{array}{l}\text { PEB- } \\
050\end{array}$ & 10.0 & 100 & 60000 & 8,415 & 0.765 & 8.34 \\
\hline $\begin{array}{l}P E B- \\
075\end{array}$ & 15.0 & 100 & 65000 & 9,116 & 0.766 & 8.39 \\
\hline $\begin{array}{r}P E B- \\
125\end{array}$ & 25.0 & 100 & 75000 & 10,519 & 0.768 & 8.47 \\
\hline $\begin{array}{l}\text { PEB- } \\
160\end{array}$ & 32.0 & 75 & 61500 & 11,500 & 0.769 & 8.22 \\
\hline $\begin{array}{r}P E B- \\
204\end{array}$ & 40.8 & 75 & 68100 & 12,735 & 0.771 & 8.00 \\
\hline $\begin{array}{l}P E B- \\
250^{b}\end{array}$ & 50.0 & 75 & 75000 & 14,025 & 0.774 & 7.76 \\
\hline
\end{tabular}

a All chains of a particular system have both the same backbone length of 500 carbons (250 monomers) and the same branch distribution (Table S.1). ${ }^{\mathrm{b}}$ Atactic Poly (1-butene) homopolymer- ${ }^{\mathrm{c}}$ Estimated at $\mathrm{T}=450 \mathrm{~K}$.

The initial structures for MD have been prepared as follows. The generation and packing of the chains were initially performed using a modified recoil growth algorithm with periodic boundary conditions [11]. As the copolymers are considered as atactic a value of $+30.5^{\circ}$ or $-30.5^{\circ}$ to the $-\mathrm{CH}_{2}-\mathrm{CH}\left(\mathrm{CH}_{2}\right)_{\mathrm{br}}-\mathrm{CH}_{2}$ - improper angle $\left(\chi_{\mathrm{ijkl}}\right)$ has been randomly assigned to the branch points (see scheme in Fig. S.1). The initial melt density was set to $0.740 \mathrm{gcm}^{-3}$ for all the systems. Subsequently, energy in the simulation box was minimized. Further equilibration was performed by 0.1 ns of NVT-MD runs and then the box volume was relaxed by 1 ns of NPT-MD runs. After the equilibration period, NPT-MD simulations were extended up to $5 \mu$ s at the temperatures 450, 509, 550 and $600 \mathrm{~K}$ using the TraPPe-UA force field [8-10]. However, two modifications of the original force field are considered: i) Instead of using, the original fixed bond model, we have applied a harmonic bond stretching potential to C-C bonds $\left(1 / 2 \mathrm{k}_{\mathrm{b}}\left(1-\mathrm{l}_{\mathrm{o}}\right)^{2}\right.$ where $\mathrm{k}_{\mathrm{b}}$ $=900 \mathrm{kcal} / \mathrm{mol}^{2}$ and $\mathrm{l}_{0}=1.54 \AA$ ) [7] and ii) in order to maintain the tacticity of the branched systems in the united atom (UA) approach, we have introduced an improper torsional angle potential in the branch points in the form of $1 / 2 \mathrm{k}_{\chi}\left(\chi_{\mathrm{ijkl}}-\chi_{\mathrm{o}}\right)^{2}$ where $\mathrm{k}_{\mathrm{b}}=123.7 \mathrm{kcal} /\left(\mathrm{mol} \mathrm{rad}^{2}\right)$ and $\chi_{0}= \pm 30.5^{\circ}$ for the two possible configurations of the branch point, see Fig. S.1) [12]. This improper dihedral potential is sufficient to keep the chiral center in its original configuration. In fact, flips between branch configurations have not been detected during the simulation.

For molecular dynamics (MD) simulations, a time step of $\Delta \mathrm{t}=2 \mathrm{fs}$ was used to integrate the motion equations using the velocity Verlet algorithm. The non-bonded interactions were calculated using the Verlet buffer cut-off scheme using a van der Waals cut-off of $1.4 \mathrm{~nm}$. The neighbor list for non-bonded interactions has been updated each 10 steps. The Nose-Hoover thermostat with a relaxation time $\left(\tau_{\mathrm{T}}\right)$ of $0.5 \mathrm{ps}$ was used to control the temperature. The pressure was set up to $1 \mathrm{~atm}$ in all NPT simulations and isotropically controlled using the ParrinelloRahman barostat with a time constant $\left(\tau_{\mathrm{P}}\right)$ for coupling of 5 ps. Cubic Periodic boundary conditions (PBC) were applied to all the three directions to remove the surface effect and to mimic the bulk state. Unless otherwise stated, the structures were dumped every 20 ps for subsequent analysis using "in-house" codes. The last $3 \mu$ s in the simulations were taken into account to calculate the ensemble averages. Molecular graphics have been generated using the VMD package [13]. All simulations were performed using GROMACS 2016.3 [14] package on GPU workstations.

\section{Results and discussion}

Testing equilibration of the models. As a measurement of the equilibration at all length scales, the orientational autocorrelation functions of the end-to-end unit vectors (ACF-EE) and the mean square internal distances, were calculated. Fig. 1 shows these functions at $450 \mathrm{~K}$ (see Figure S.2 and S. 3 for the rest of temperatures). The ACF-EE functions decay to zero for all systems and temperatures indicating the full relaxation of the polymer chains and the loss of the memory of its initial conformations (see Fig. 1 and S.2). As expected, the ACF-EE decay is greater as the temperature increases for all the systems under study. Also, the value of the relaxation of the full chain is slower as the number of branches increases. Furthermore, smooth curves are observed for all the mean square internal distances in all simulations without chain deformations, indicating a good equilibration of internal segments on short and intermediate scales (Fig. S.3).

Densities and thermal expansion coefficient. Calculated densities show a very good agreement with experimental values. In Fig S.4.a, melt densities at $450 \mathrm{~K}$ (listed in Table 1 ) as a function of the SCB content are compared to the experimental results obtained from the literature.

The thermal expansion coefficient, $\alpha=-d \ln \rho / d T=-\frac{1}{\rho} d \rho / d T$, can be extracted from the temperature dependence of the simulated density values (Fig S.4.b). For linear high molecular weight PE and poly(1butene) the experimental values of $\alpha$ vary between 7.6-8.8 $\times 10^{-4} \mathrm{~K}^{-1}$ and 7.4-7.6 $\times 10^{-4} \mathrm{~K}^{-1}$ at $439 \mathrm{~K}$, respectively $[5,15]$. These experimental values are in nice agreement with our simulations (see Table 1). Furthermore, the experimental $\alpha$ results for polyolefins with

different type of branches are practically the same across the whole range of comonomer content in Ziegler-Natta and single site polyolefins, which suggests that SCB does not significantly affect the value of $\alpha$ [15]. Our simulations also show that the $\alpha$ values are not affected for branch content lower that 25 branches/100 backbone carbons, within the range of branches experimentally reported in Ref. [15]. For contents greater than 25 branches $/ 100 \mathrm{C}$, the $\alpha$ value shows a clear decreasing aspect.

Chain Dimensions. Molecular dimensions obtained for the systems studied are listed in Table S.2. It is worthwhile to mention that from the simulated values of $\left\langle\mathrm{R}_{0}^{2}\right\rangle$ and $\left\langle\mathrm{R}_{\mathrm{g}}^{2}\right\rangle$, the Flory random coil approach $\left\langle\mathrm{R}_{0}^{2}\right\rangle$ $=6\left\langle\mathrm{R}_{\mathrm{g}}^{2}\right\rangle$ nicely fulfills for all cases (see Fig. S.5 in the Supplementary Information). The results indicate that for chains of equal length (500 C atoms), the chain dimensions are reduced as the concentration of SCB increases at all temperatures between 450 and $600 \mathrm{~K}$. This general behavior has been obtained previously in a more limited SCB content range at $450 \mathrm{~K}$ using Monte Carlo simulations [5,18].

The molecular weight invariant $\left\langle\mathrm{R}_{\mathrm{g}}^{2}\right\rangle / \mathrm{M}$, can be obtained for all the models and the results are compared to those obtained experimentally by SANS in the whole range of SCB content up to poly (1-butene). The experiments show a monotonic decrease of $\left\langle\mathrm{R}_{\mathrm{g}}^{2}\right\rangle / \mathrm{M}$ with branching within a temperature range of 300-440 K [6]. Fig S. 6 shows the comparison of the experimental values obtained for $\left\langle\mathrm{Rg}^{2}\right\rangle / \mathrm{M}$ at $440 \mathrm{~K}$ with those obtained in our simulations at $450 \mathrm{~K}$. Clearly, the chain dimension decreases as the number of ethyl branches increases. In general, the results compare quite well with those obtained by SANS experiments [6]. Also a reasonable agreement is found with previous Monte Carlo 

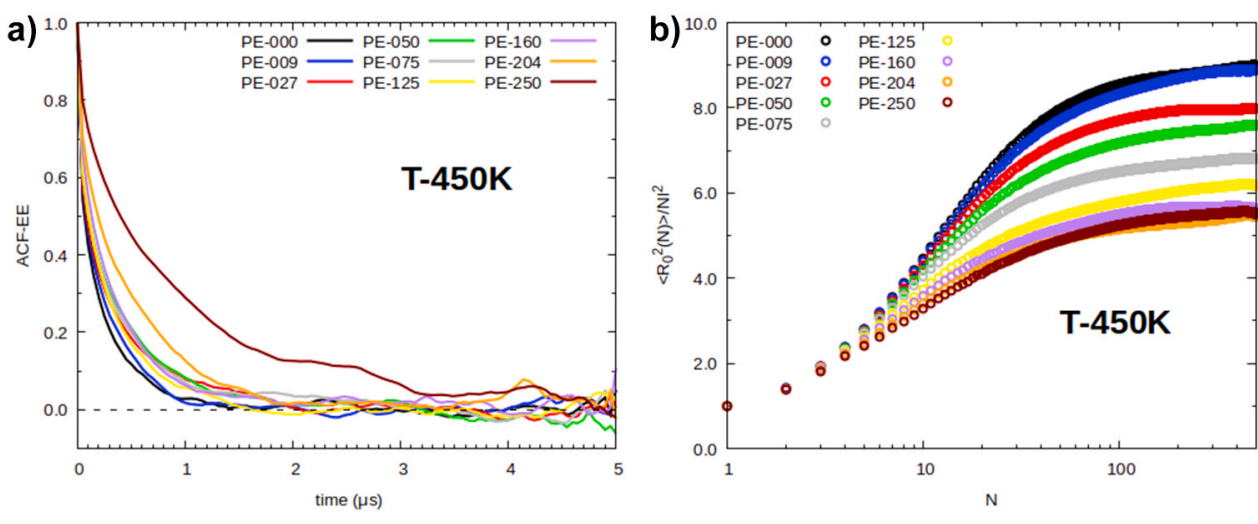

Fig. 1. a) Orientational autocorrelation functions of the end-to-end unit vectors (ACF-EE) for all systems at 450K. ACF-EE is defined as $<\boldsymbol{R}_{e e}(t) \boldsymbol{R}_{e e}(0)>/<\boldsymbol{R}_{e e}^{2}>$, where $\boldsymbol{R}_{e e}(t)$ is the end-to-end distance at time t. The decay of this function to zero is a measure of the rate of full relaxation of the polymer chain [16]. and b) Mean square internal distance $\left\langle\boldsymbol{R}^{2}(\boldsymbol{N})\right\rangle /<$ $N l^{2}>$ as a function of $\mathrm{N}$, where $\mathrm{N}$ is the distance between the monomer $\mathrm{jth}$ and the $\mathrm{j}^{\mathrm{th}}+$ $\mathrm{N})$ monomer and 1 stands for the bond distance (1.54 ̊). This function is used to understand the correlation between internal monomers in a chain. A smooth curve is observed for all systems and temperatures, no chain deformations on intermediate scales are observed which can be interpreted as a well-equilibrated system. More information about this function

can be found in Ref. [17].

(MC) simulations using the same force field [18] and results obtained by Neelakantan et al. in MD simulations of united atom models of EB copolymers at $\mathrm{T}=423 \mathrm{~K}$ using the OPLS-UA force field [19].

In the literature, some recent attempts have been made to study the effect of temperature on the chain dimensions for selected homopolymers as polyethylene, polypropylene, polystyrene and polydimethylsiloxane [20] and ethylene-propylene copolymers of different tacticity [21]. In the case of linear PE, MC [22] and MD simulations [7] have been reported within a broad temperature range $300-600 \mathrm{~K}$. In this work, we have extended this study to ethylene-butene copolymers from linear to high contents of ethyl branches.

The key property at this respect is the temperature dependence on macromolecular dimensions, $\kappa=d \ln R_{g}^{2} / d T$, which has a significant role on entanglement features [23]. Herein, the agreement between simulated $\left(-1.10 \times 10^{-3} \mathrm{~K}^{-1}\right)$ and experimental $\left(-1.06\right.$ to $\left.-1.25 \times 10^{-3} \mathrm{~K}^{-1}\right)$ $\kappa$ values is quite good in the case of linear PE (PEB-000) $[5,7,22]$.

In Fig. 2 a the $\ln \left\langle\mathrm{R}_{\mathrm{g}}^{2}\right\rangle$ at different temperatures for PEB-000, PEB-160 and PEB-250 are displayed (see Fig. S.7 for all studied systems). Values of $\kappa$ as a function of the content branch have been extracted from the slope of the linear fitted lines. It is quite interesting the behavior obtained with increasing branching, which goes from negative to positive $\kappa$ values. It is clearly seen that the value of $\kappa=0$ is obtained close to an ethyl branch content of around 25-30 every 100 carbon atoms. The trend observed in our simulations is in perfect agreement with the values of $\kappa$ measured by SANS for ethylene/1-butene copolymers [6] and poly (1-butene) [24] as it can be observed in Fig. 2 b, where both simulated and experimental values are compared.

The decrease of coil dimensions with temperature (negative $\kappa$ ) has been suggested to be due to a decrease of the trans population of dihedral angles with increasing temperature. Fetters et al. argued that the addition of ethyl branches to the main chain reverses the trans/gauche population when compared to the linear PE, in which the gauche conformers have a lower probability than the trans conformers [6]. This reversing would lead to increased values of $\kappa$ with branching content.

RIS calculations on unperturbed isolated chains to study the conformational properties of polymeric chains, including ethyl branched polyolefins, are available in the literature [25,26]. These RIS calculations show a monotonic decrease in the chain dimensions of PEB as increase the number of 1-butene units, in qualitative agreement with both the experimental measurements and the MD simulations here reported. In previous MC simulations performed on ethylene/1-hexene copolymers, it was concluded that the decrease observed in the coil dimensions as the amount of SCB increases goes parallel to an increase of the population of backbone gauche conformers [11]. Furthermore, RIS calculations seem to show that sparsely branched polymers (20 or more bonds apart) vary insignificantly from linear polyethylene. In the current MD simulations, the $\left\langle\mathrm{R}_{\mathrm{g}}^{2}\right\rangle$ of the less branched polymers is reduced only in 3\% for PEB-009 (branches spaced 49 bonds apart) with respect to the linear polyethylene (PEB-000). In the case of PEB-027 (branches spaced 17 bonds apart) the reduction is more significant (around 7-12\%).

To examine how both the number of ethyl branches and temperature affect the chain conformation of PEB systems, an analysis of the torsion angle distribution along the chains has been carried out. The evolution of the relative population of different chain dihedral states extracted from the MD simulations for PEB-000, PEB-125 and PEB-250 as a function of the temperature are shown in Fig. 3.

In the case of PEB-000 the relative population of trans ( $t$ ) states and trans-trans dyads (tt) clearly decreases as temperature increases. Furthermore, these are the most populated states at the different temperatures. Consequently, the relative population of gauche $\left(\mathrm{g}^{+}, \mathrm{g}^{-}\right)$ conformers increases, an increment also observable in $\mathrm{tg}^{+}, \mathrm{g}^{+} \mathrm{g}^{+}, \mathrm{tg}^{-}$and $\mathrm{g}^{-} \mathrm{g}^{-}$dyads. It should be noted that the distance between the end atoms of two consecutive monomeric units decreases following the order $\mathrm{tt}>\mathrm{t}$ $\mathrm{g}^{+}, \mathrm{t} \mathrm{g}^{-}>\mathrm{g}^{+} \mathrm{g}^{+}, \mathrm{g}^{-} \mathrm{g}^{-}$, as depicted in Fig S.9. Obviously, the enlarged population of gauche states as temperature increases lead to the contraction of the coils and, therefore, a negative value of $\kappa$.

The PEB-160 system has a $\kappa$ value positive, that is the size of the chain increases as the temperature increases. As it can be seen in Fig. 3 (and Fig. S.8), the population of $\mathrm{tg}^{+} / \mathrm{g}^{+} \mathrm{t}$ and $\mathrm{tg}^{-} / \mathrm{g}^{-} \mathrm{t}$ are most populated than the tt states in the whole range of temperatures. Although, a decrease in $\mathrm{tt}$ states is observed as $\mathrm{T}$ increases, the trend of $\mathrm{tg}^{+} / \mathrm{g}^{+} \mathrm{t}$ and $\mathrm{tg}^{-} / \mathrm{g}^{-} \mathrm{t}$ states are reversed as compared with the PEB-000 system (Fig. 3. c). This should explain the small positive value of $\kappa$ observed in this system.

The PEB-250 model represents the other compositional extreme and it is also shown in Fig. 3. Interestingly, there is no appreciable change with temperature in the relative population of $\mathrm{t}, \mathrm{g}^{+}$and $\mathrm{g}^{-}$conformers (Fig. 3.c). In contrast dyad states are clearly affected for the temperature. Again, most populated $\mathrm{tg}^{+} / \mathrm{g}^{+} \mathrm{t}$ and $\mathrm{tg}^{-} / \mathrm{g}^{-} \mathrm{t}$ states are observed. Furthermore, in this case, as temperature increases the relative population of $\mathrm{tt}$ conformers increases at the cost of $\mathrm{tg}^{+}$and $\mathrm{tg}^{-}$conformers, but $\mathrm{g}^{+} \mathrm{g}^{+}$and $\mathrm{g}^{-} \mathrm{g}$ conformers still remain unchanged with temperature. As a result, this variation gives rise to the increase of chain dimensions and clear positive value of $\kappa$ as temperature increases.

The results obtained in the complete set of simulations suggest that monotonic decrease of tt population causes the decreased chain dimensions as branching increases, as observed in Fig. 1. Conversely, a change in the temperature trend of the relative population of $\mathrm{tt} \mathrm{tg}^{+}$and $\operatorname{tg}^{-}$conformers causes the change from negative to positive $\kappa$ as branching increases shown in Fig. 2.

These analysis give a suitable physical explanation to the experimental observations [6] regarding the change of sign for the 

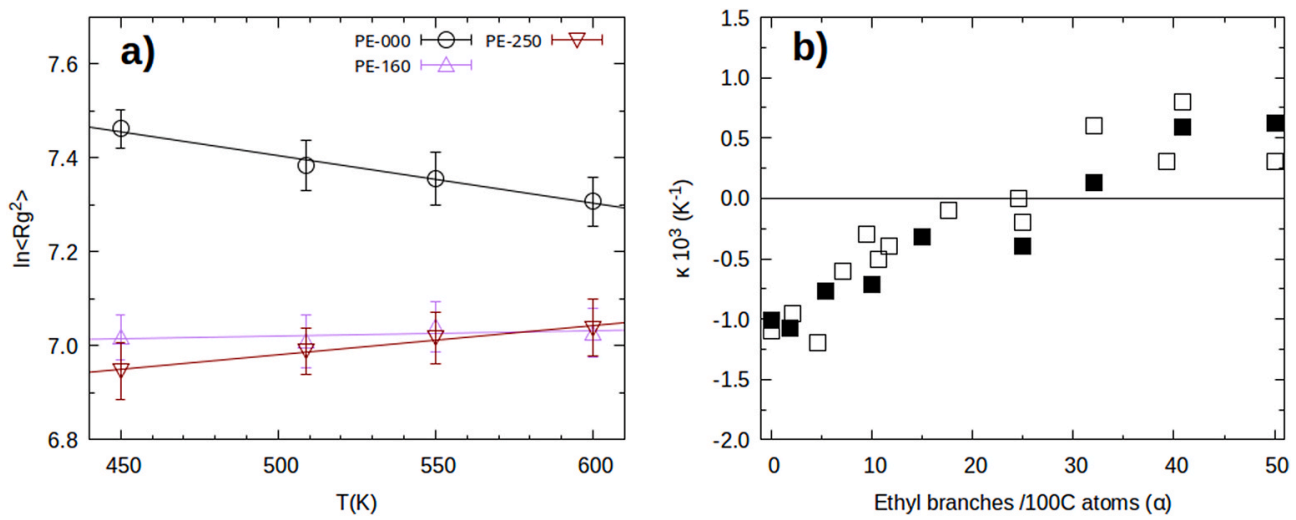

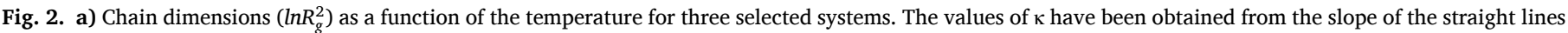

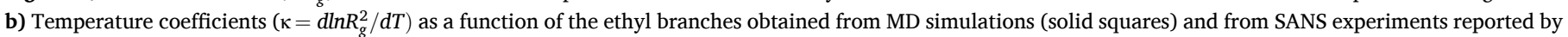
Fetters et al. for PEB copolymers [6] and Zirkel et al. for poly(1-butene) [24] (open squares).

a)
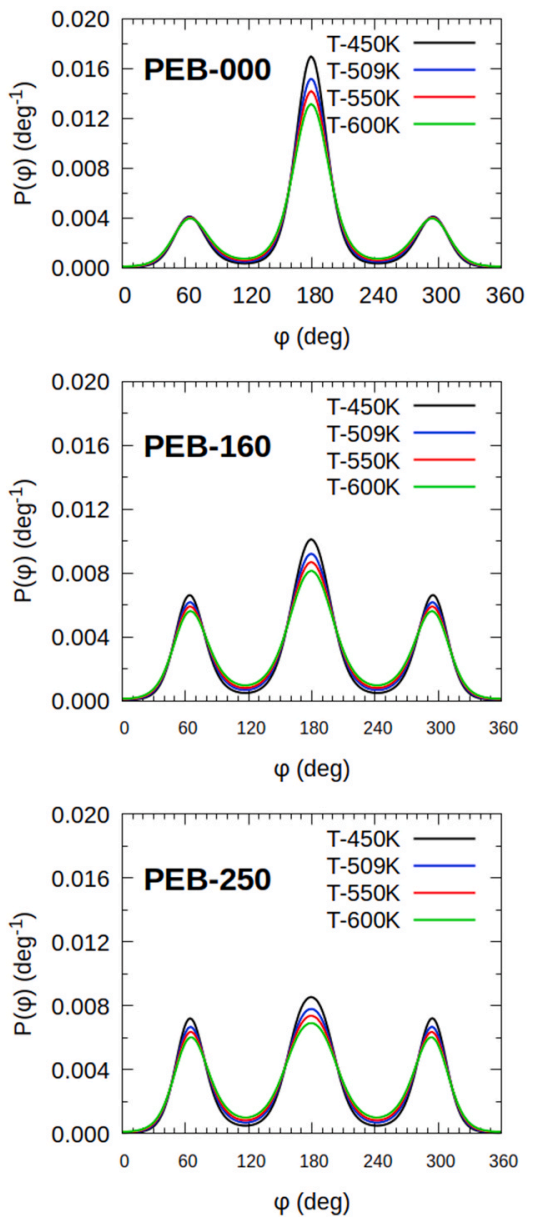

b)
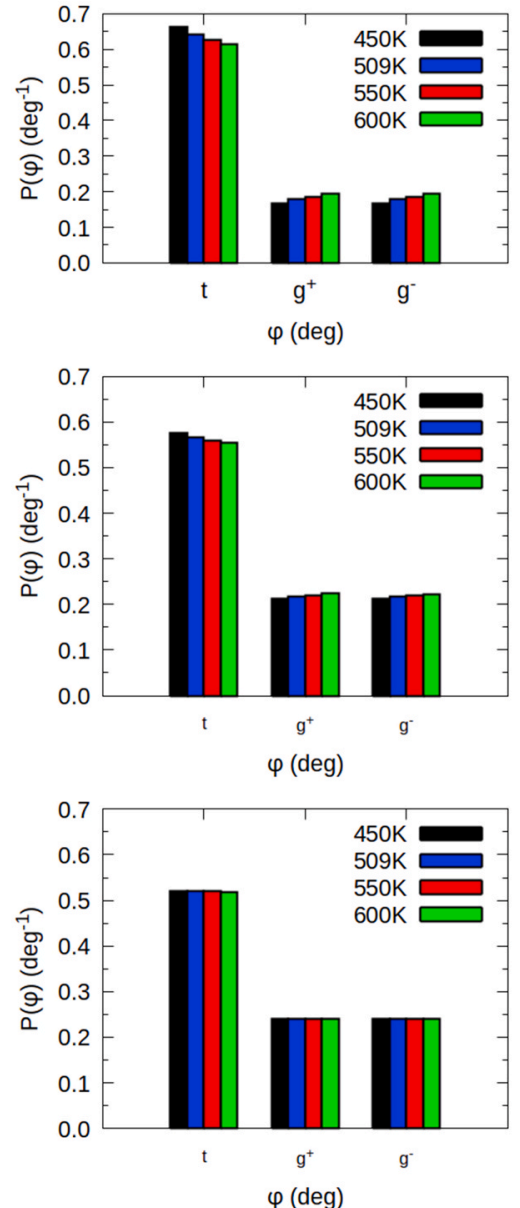

c)
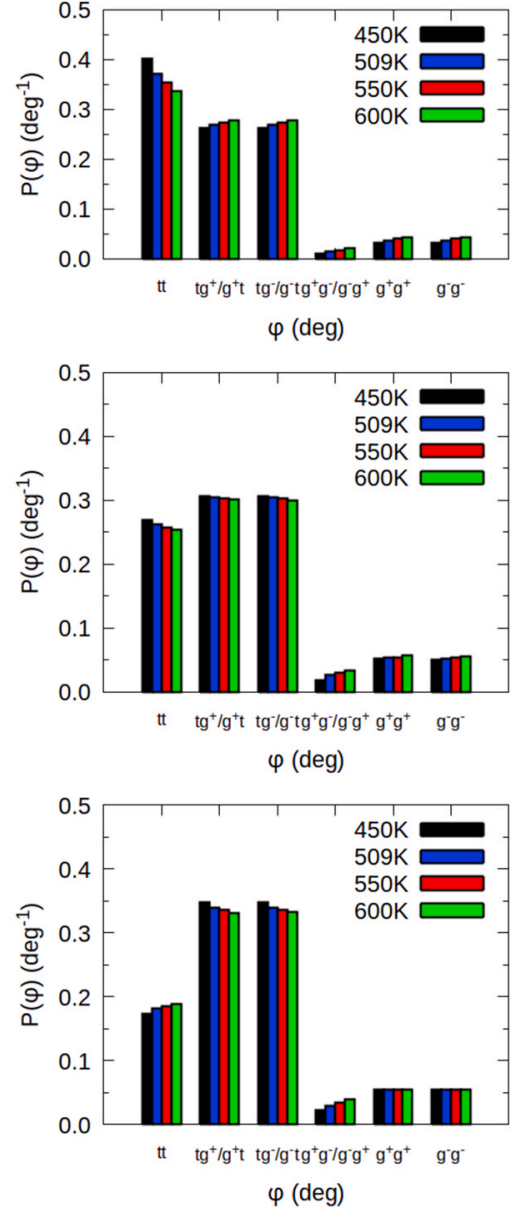

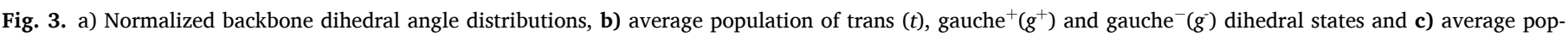

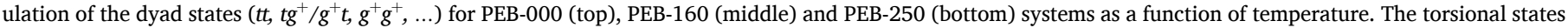
are defined as follows: $g^{-}$state corresponds to a torsional angle in the range $0^{\circ} \leq \phi \leq 120^{\circ}, t$ state to $120^{\circ} \leq \phi \leq 240^{\circ}$ and $g^{+}$state to $240^{\circ} \leq \phi \leq 360^{\circ}$.

temperature dependence of macromolecular dimension $(\kappa)$ as the number of ethyl branches increases on the backbone polymer chain as a function of the temperature.

\section{Conclusion}

We have performed MD simulations on a set of ethylene/1-butene models with different branching content from linear polyethylene to poly(1-butene) over a broad range of temperature in order to determine the characteristic macromolecular sizes. We have found that the $\left\langle\mathrm{R}_{\mathrm{g}}^{2}\right\rangle / \mathrm{M}$ 
ratio decreased with increasing ethyl branch concentration, as observed in previous works. In addition, we have found that the temperature dependence of macromolecular dimension ( $\kappa$ ) changes from negative to positive as the ethyl branch concentration increases. Both results are in perfect agreement with the SANS experiments performed by Fetters et al., in 1997 in hydrogenated polybutadiene. A systematic study of the population of conformers in the model systems indicates that the decrease observed in coil dimensions as SCB increases at a given temperature goes parallel to the increase of the relative population of gauche conformers. Below 25-30 ethyl branches every 100 carbon atoms, negative values of $\kappa$ have been obtained, which is related to an increase population of the dense gauche conformation with temperature. Notwithstanding, the situation reverses with SCB content higher than 25-30 ethyl branches every 100 carbon atoms. In these case the increase of the temperature favors the more extended trans conformers, giving rise to positive values of $\kappa$. Thus, the results shown by SANS in 1997, and now computer simulations in the current manuscript, show that there exists a strong influence of the frequency of side groups on the temperature dependence connected with the different population of trans/ gauche states of the main chain. This behavior was not predicted via RIS theory. Additionally this trend with the temperature has not been explained before neither theoretically nor by computer simulation.

\section{Funding sources}

Ministerio de Ciencia, Innovación y Universidades (MICIU), grant number PID2019-107710GB-I00.

\section{Declaration of competing interest}

The authors declare that they have no known competing financial interests or personal relationships that could have appeared to influence the work reported in this paper.

\section{Acknowkledgments}

The authors thank to the MICIU (Ministerio de Ciencia, Innovación y Universidades), grant number PID2019-107710GB-I00, for the financial support of this investigation.

\section{Appendix A. Supplementary data}

Supplementary data to this article can be found online at https://doi. org/10.1016/j.polymer.2021.123772.

\section{References}

[1] L.J. Fetters, D.J. Lohse, S.T. Milner, W.W. Graessley, Packing length influence in linear polymer melts on the entanglement, critical, and reptation molecular weights, Macromolecules 32 (1999) 6847-6851, https://doi.org/10.1021/ ma990620o.

[2] L.J. Fetters, D.J. Lohse, C.A. García-Franco, P. Brant, D. Richter, Prediction of melt state poly $(\alpha$-olefin) rheological properties: the unsuspected role of the average molecular weight per backbone bond, Macromolecules 35 (2002) 10096-10101, https://doi.org/10.1021/ma025659z.

[3] J.F. Vega, J. Ramos, J. Martínez-Salazar, Effect of short chain branching in molecular dimensions and Newtonian viscosity of ethylene/1-hexene copolymers: matching conformational and rheological experimental properties and atomistic simulations, Rheol. Acta 53 (2014) 1-13, https://doi.org/10.1007/s00397-0130735-1.

[4] H.J. Unidad, M.A. Goad, A.R. Bras, M. Zamponi, R. Faust, J. Allgaier, W. PyckhoutHintzen, A. Wischnewski, D. Richter, L.J. Fetters, Consequences of increasing packing length on the dynamics of polymer melts, Macromolecules 48 (2015) 6638-6645, https://doi.org/10.1021/acs.macromol.5b00341.

[5] J. Ramos, J.F. Vega, J. Martínez-Salazar, Predicting experimental results for polyethylene by computer simulation, Eur. Polym. J. 99 (2018) 298-331, https:// doi.org/10.1016/j.eurpolymj.2017.12.027.

[6] L.J. Fetters, W.W. Graessley, R. Krishnamoorti, D.J. Lohse, Melt chain dimensions of poly(ethylene-1-butene) copolymers via small angle neutron scattering, Macromolecules 30 (1997) 4973-4977, https://doi.org/10.1021/ma961408c.

[7] J. Ramos, J.F. Vega, J. Martínez-Salazar, Molecular dynamics simulations for the description of experimental molecular conformation, melt dynamics, and phase transitions in polyethylene, Macromolecules 48 (2015) 5016-5027, https://doi. org/10.1021/acs.macromol.5b00823.

[8] M.G. Martin, J.I. Siepmann, Transferable potentials for phase equilibria. 1. Unitedatom description of n-alkanes, J. Phys. Chem. B 102 (1998) 2569-2577, https:// doi.org/10.1021/jp972543+.

[9] M.G. Martin, J.I. Siepmann, Novel configurational-bias Monte Carlo method for branched molecules. Transferable potentials for phase equilibria. 2. United-atom description of branched alkanes, J. Phys. Chem. B 103 (1999) 4508-4517, https:// doi.org/10.1021/jp984742e.

[10] K.A. Maerzke, J.I. Siepmann, Transferable potentials for phase equilibria - coarseGrain description for linear alkanes, J. Phys. Chem. B 115 (2011) 3452-3465, https://doi.org/10.1021/jp1063935.

[11] J. Ramos, L.D. Peristeras, D.N. Theodorou, Monte Carlo simulation of short chain branched polyolefins in the molten state, Macromolecules 40 (2007) 9640-9650, https://doi.org/10.1021/ma071615k.

[12] D. Heine, D.T. Wu, J.G. Curro, G.S. Grest, Role of intramolecular energy on polyolefin miscibility: isotactic polypropylene/polyethylene blends, J. Chem. Phys. 118 (2003) 914-924, https://doi.org/10.1063/1.1516591.

[13] W. Humphrey, A. Dalke, K. Schulten, VMD: visual molecular dynamics, J. Mol. Graph. 14 (1996) 33-38, https://doi.org/10.1016/0263-7855(96)00018-5.

[14] M.J. Abraham, T. Murtola, R. Schulz, S. Páll, J.C. Smith, B. Hess, E. Lindah, Gromacs: high performance molecular simulations through multi-level parallelism from laptops to supercomputers, SoftwareX 1-2 (2015) 19-25, https://doi.org/ 10.1016/j.softx.2015.06.001.

[15] S.J. Han, D.J. Lohse, P.D. Condo, L.H. Sperling, Pressure-volume-temperature properties of polyolefin liquids and their melt miscibility, J. Polym. Sci., Part B: Polym. Phys. 37 (1999) 2835-2844, https://doi.org/10.1002/(SICI)1099-0488 (19991015)37:20<2835::AID-POLB3 > 3.0.CO, 2-1.

[16] M. Doi, S.F. Edwards, S.F. Edwards, The Theory of Polymer Dynamics, Clarendon Press, 1988.

[17] R. Auhl, R. Everaers, G.S. Grest, K. Kremer, S.J. Plimpton, Equilibration of long chain polymer melts in computer simulations, J. Chem. Phys. 119 (2003) 12718-12728, https://doi.org/10.1063/1.1628670.

[18] K. Moorthi, K. Kamio, J. Ramos, D.N. Theodorou, Monte Carlo simulation of short chain branched polyolefins: structure and properties, Macromolecules 45 (2012) 8453-8466, https://doi.org/10.1021/ma301322v.

[19] A. Neelakantan, R. Stine, J.K. Maranas, Chain packing in ethylene-butene copolymers, Macromolecules 36 (2003) 3721-3731, https://doi.org/10.1021/ ma0210729.

[20] P.N. Tzounis, S.D. Anogiannakis, D.N. Theodorou, General methodology for estimating the stiffness of polymer chains from their chemical constitution: a single unperturbed chain Monte Carlo algorithm, Macromolecules 50 (2017) 4575-4587, https://doi.org/10.1021/acs.macromol.7b00645.

[21] P.N. Tzounis, D.V. Argyropoulou, S.D. Anogiannakis, D.N. Theodorou, Tacticity effect on the conformational properties of polypropylene and poly(ethylenepropylene) copolymers, Macromolecules 51 (2018) 6878-6891, https://doi.org/ 10.1021/acs.macromol.8b01099.

[22] K. Foteinopoulou, N.C. Karayiannis, M. Laso, M. Kröger, Structure, dimensions, and entanglement statistics of long linear polyethylene chains, J. Phys. Chem. B 113 (2009) 442-455, https://doi.org/10.1021/jp808287s.

[23] J.M. Carella, W.W. Graessley, L.J. Fetters, Effects of chain microstructure on the viscoelastic properties of linear polymer melts: polybutadienes and hydrogenated polybutadienes, Macromolecules 17 (1984) 2775-2786, https://doi.org/10.1021/ ma00142a059.

[24] A. Zirkel, V. Urban, D. Richter, L.J. Fetters, J.S. Huang, R. Kampmann, N. Hadjichristidis, Small-angle neutron scattering evaluation of the temperature dependence of atactic polypropylene and poly(1-butene) chain dimensions in the melt, Macromolecules 25 (1992) 6148-6155, https://doi.org/10.1021/ ma00049a010.

[25] A.E. Tonelli, Conformational characteristics and flexibility of branched polyethylenes, J. Am. Chem. Soc. 94 (1972) 2972-2979, https://doi.org/10.1021/ ja00764a013.

[26] W.L. Mattice, Reduction of the unperturbed dimensions of the main chain when ethyl groups are attached to a poly(methylene) backbone, Macromolecules 19 (1986) 2303-2305, https://doi.org/10.1021/ma00162a034. 